\title{
Effects of an acute $\alpha$-lactalbumin manipulation on mood and food hedonics in high- and low-trait anxiety individuals
}

\author{
Ellen Verschoor ${ }^{1}$, Graham Finlayson ${ }^{2}$, John Blundell ${ }^{2}$, C. Rob Markus ${ }^{1}$ and Neil A. King ${ }^{3}$ \\ ${ }^{1}$ Department of Neuropsychology and Psychopharmacology, Faculty of Psychology and Neuroscience, Maastricht University, \\ PO Box 616, 6200 MD Maastricht, The Netherlands \\ ${ }^{2}$ Biopsychology Group, Institute of Psychological Sciences, University of Leeds, Leeds, West Yorkshire, LS2 9JT, UK \\ ${ }^{3}$ Institute of Health and Biomedical Innovation, Queensland University of Technology, Brisbane 4059 QLD, Australia \\ (Received 4 September 2009 - Revised 11 February 2010 - Accepted 12 February 2010 - First published online 23 March 2010)
}

Serotonergic hypofunction is associated with a depressive mood state, an increased drive to eat and preference for sweet (SW) foods. High-trait anxiety individuals are characterised by a functional shortage of serotonin during stress, which in turn increases their susceptibility to experience a negative mood and an increased drive for SW foods. The present study examined whether an acute dietary manipulation, intended to increase circulating serotonin levels, alleviated the detrimental effects of a stress-inducing task on subjective appetite and mood sensations, and preference for SW foods in high-trait anxiety individuals. Thirteen high- (eleven females and two males; anxiety scores 45.5 (SD 5.9); BMI 22.9 (SD 3.0) $\mathrm{kg} / \mathrm{m}^{2}$ ) and twelve low- (ten females and two males; anxiety scores 30.4 (SD 4.8); BMI 23.4 (SD 2.5) $\mathrm{kg} / \mathrm{m}^{2}$ ) trait anxiety individuals participated in a placebo-controlled, two-way crossover design. Participants were provided with $40 \mathrm{~g} \alpha$-lactalbumin (LAC; L-tryptophan (Trp):large neutral amino acids (LNAA) ratio of 7.6) and $40 \mathrm{~g}$ casein (placebo) (Trp:LNAA ratio of 4.0) in the form of a snack and lunch on two test days. On both the test days, participants completed a stress-inducing task $2 \mathrm{~h}$ after the lunch. Mood and appetite were assessed using visual analogue scales. Changes in food hedonics for different taste and nutrient combinations were assessed using a computer task. The results demonstrated that the LAC manipulation did not exert any immediate effects on mood or appetite. However, LAC did have an effect on food hedonics in individuals with high-trait anxiety after acute stress. These individuals expressed a lower liking $(P=0.012)$ and SW food preference $(P=0 \cdot 014)$ after the stressful task when supplemented with LAC.

Serotonin: Trait anxiety: Stress: $\alpha$-Lactalbumin: Mood: Food hedonics

The importance of brain serotonin (5-hydroxytryptamine, $5-\mathrm{HT})$ in the regulation of mood and eating behaviour has been demonstrated by pharmacological studies. Increasing 5-HT via serotonergic (5-HTergic) agonists elevates positive $\operatorname{mood}^{(1-4)}$, and reduces motivation to eat ${ }^{(5,6)}$ and sweet (SW) food consumption ${ }^{(7)}$. Conversely, diminishing the neurotransmitter via 5-HT antagonists induces a depressive mood state $^{(8)}$ and an increased carbohydrate-rich food intake ${ }^{(9,10)}$.

Besides pharmacological studies, the acute tryptophan depletion (ATD) paradigm has been used to investigate the function of 5-HTergic neurotransmission in mood and eating behaviour. ATD induces a temporary global reduction of 5-HT synthesis in the brain by decreasing the availability of its precursor L-tryptophan (TRP) ${ }^{(11)}$. Although ATD induces a lowering in mood and an increased SW food intake, these effects are particularly, or sometimes solely, observed in individuals with abnormalities in 5-HTergic functioning ${ }^{(12-14)}$. For instance, studies show that remitted depressed patients relapsed into a depressive episode after ATD, while the mood of healthy participants was not affected ${ }^{(15,16)}$. In another study, ATD induced an increased food intake in women suffering from bulimia nervosa, whereas food intake of healthy, non-bulimic women remained stable ${ }^{(17)}$. Consequently, insights from ATD studies gave rise to the term 'serotonergic vulnerability' - used to describe the findings that some individuals have a 5-HTergic system that is more vulnerable to 5-HTergic alterations probably due to a priori abnormalities in the functioning of this system ${ }^{(18,19)}$.

Although deficiencies in the 5-HTergic system are mainly associated with clinical syndromes, it has been suggested that 5-HTergic vulnerability may also occur in healthy individuals with chronic stress experiences, which increases susceptibility to depressogenic effects of acute stress exposure $^{(20)}$. Overall, acute stress causes an increase in cortisol and 5-HT as important biological conditions enabling the human brain to cope with stress. Nevertheless, chronic stressed or stress-prone individuals perceive many events as stressful, and will therefore frequently experience high cortisol

Abbreviations: 5-HT, 5-hydroxytryptamine; 5-HTergic, 5-HT via serotonergic; ATD, acute tryptophan depletion; HA, high-anxiety; LA, low-anxiety; LAC, $\alpha$-lactalbumin; LNAA, large neutral amino acids; NA, negative affects; PA, positive affects; SA, savoury; STAI, State-Trait Anxiety Inventory; SW, sweet; TRP, L-tryptophan.

* Corresponding author: Ellen Verschoor, fax +3143 3884560, email ellen.verschoor@maastrichtuniversity.nl 
levels. In the long term, chronic hypersecretion of cortisol results in decreased 5-HT receptor sensitivity and diminution of 5-HT turnover ${ }^{(21)}$. It is proposed that the ensuing functional shortage of 5-HT during stress may cause individuals to become more sensitive to stressors, and hence to be particularly susceptible to debilitated mood ${ }^{(22)}$, an increased drive to eat and an increased desire for SW foods ${ }^{(23-25)}$ during acute exposure to stress. Moreover, it has been proposed that the detrimental effects of stress on mood and eating behaviour in stress-susceptible individuals could be moderated by an increase in 5-HT. Consistent with this premise are a series of studies conducted by Markus et al. ${ }^{(26-28)}$. This research group used $\alpha$-lactalbumin (LAC), a whey-derived protein with the highest TRP concentration of all protein fractions ${ }^{(29)}$, to increase the plasma ratio of TRP to the other large neutral amino acids (LNAA). This increases transport of TRP across the blood-brain barrier, thereby enhancing brain 5-HT synthesis and potentially 5-HT neurotransmission ${ }^{(30)}$. In accordance with the hypotheses, LAC reduced prolactin and cortisol concentrations and improved mood during a stressful task in stress-prone individuals ${ }^{(26,27)}$. Yet, a similar study by Merens et al. ${ }^{(31)}$ did not replicate this effect in recovered depressed patients. Furthermore, a study investigating the effects of LAC on eating behaviour found no effect of the intervention on subjective appetite, macronutrient preference and food intake ${ }^{(32)}$. However, in the present study, information about the susceptibility to stress was not measured.

The aim of the present study was to examine the acute effects of LAC on mood, appetite and food hedonics (liking and food preference) in individuals susceptible to stress before and after exposure to an acute stress task. It was hypothesised that (1) any detrimental effects of acute stress on mood would be alleviated by acute administration of LAC in high-trait individuals; and (2) acute administration of LAC would reduce the stress-induced preference for SW foods in high-trait individuals.

\section{Experimental methods}

\section{Participants}

Australian University students were emailed to complete an online screening questionnaire package containing the State-Trait Anxiety Inventory (STAI) ${ }^{(33)}$, and a questionnaire concerning personal details. The STAI consists of two separate self-reported subscales: the trait anxiety scale and the state anxiety scale. The trait anxiety scale refers to a stable tendency to experience anxiety and to perceive stressful situations as threatening ${ }^{(34)}$, and thereby refers to chronic feelings of anxiety and/or stress. Of the 108 email respondents, twenty-six students met the eligibility criteria, and expressed an interest in participating in the study. A further $76 \%$ of email respondents met the eligibility criteria, but declined due to time constraints preventing their participation. Furthermore, one participant did not complete the study (withdrew before the second session) for reasons unrelated to treatment. Means and standard deviations age of the twenty-five participants who completed the study was 21.3 (SD 4.2) years. Exclusion criteria for participation were a BMI $>30 \cdot 0 \mathrm{~kg} / \mathrm{m}^{2}$, chronic or current physical or psychiatric illness, medication use, irregular diets or deviant eating habits, smoking, excessive use of alcohol $(>20$ units a week), coffee ( $>10$ cups a day) or other drugs, and allergy for milk products. Participants were separated into two groups according to a median split in their trait anxiety scores of the STAI, resulting in a high-anxiety (HA) group of thirteen participants (eleven women and two men, STAI scores 45.5 (SD 5.9)) and a low-anxiety (LA) group of twelve participants (ten women and two men, STAI scores 30.4 (SD 4.8)). Previous research has shown that the mean score on the STAI of healthy volunteers is 33.39 (SD 13.51), while individuals' scores with a panic disorder, social phobia and specific phobia ranged between $47 \cdot 39$ (SD 13.51) and $55.93(\mathrm{SD} 8 \cdot 30)^{(35)}$. All participants had a BMI in the range of $20-28 \mathrm{~kg} / \mathrm{m}^{2}(22.9$ (SD 3.0$)$ in the HA group and 23.4 (SD 2.5) in the LA group). The present study was conducted according to the guidelines laid down in the Declaration of Helsinki, and all procedures were approved by the ethics committee of the Queensland University of Technology. Written informed consent was obtained from all participants before participation.

\section{Design and procedure}

The study conformed to a placebo-controlled, two-way crossover design. All participants underwent two experimental test days, which differed by the type of dietary manipulation. The two conditions were LAC and casein placebo (CAS). The intervention order was counterbalanced over the two test days with a washout period of 1 week.

Participants were instructed not to drink alcohol or take part in vigorous physical activity for $24 \mathrm{~h}$ before the sessions. Participants were required to consume their regular breakfast at home by 07.30 hours. In addition, volunteers were requested to eat a similar breakfast on both test days in order to ensure comparable baseline conditions. Fig. 1 shows a schematic diagram of the experimental protocol. On arrival at the laboratory at 09.00 hours, participants completed a questionnaire measuring their previous night's sleep quality and compliance to the dietary instructions. Thereafter, participants completed a 20-min test battery including baseline measurements of mood, appetite and food hedonics. Then, participants were provided with a snack and the LAC or placebo chocolate drink. Directly after intake, a palatability questionnaire was completed followed by a 90 -min rest period. After this rest period, participants completed a second test battery session including comparable measurements of mood, appetite and food hedonics. Immediately after the second test battery,

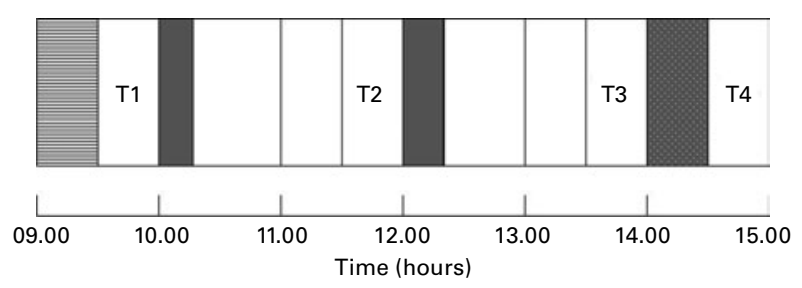

Fig. 1. A schematic diagram to show the temporal profile of events and measurements during the test day. T1, Test block 1 (baseline); T2, test block 2 (after snack); T3, test block 3 (after lunch); T4, test block 4 (after stress). 自, Arrival; $\square$, test meal; $\square$, stress task. 
participants were provided with a lunch including a second LAC or placebo chocolate drink, and they subsequently completed a post-lunch palatability questionnaire. Ninety minutes after the lunch, a third (pre-stress) test battery was completed followed by the stress task. Immediately after the stress task, a fourth (post-stress) test battery was completed.

\section{Dietary manipulation}

During both the experimental test days, participants received a snack, lunch and two chocolate drinks that provided a total energy intake of $4229.09 \mathrm{~kJ}$, of which $9 \%$ energy was from fat, $73 \%$ energy from carbohydrate and $18 \%$ energy from protein. The snack consisted of biscuits $(665.70 \mathrm{~kJ})$ with one chocolate drink $(697.94 \mathrm{~kJ})$, whereas lunch consisted of two slices of bread with ham and jam and a yoghurt $(2167.51 \mathrm{~kJ})$, including an additional chocolate drink $(697.94 \mathrm{~kJ})$.

During both the days, the foods were equienergetic and similar in appearance, taste and macronutrient content. The only characteristic which varied between days was the protein composition of the chocolate drink. In the LAC condition, each chocolate drink contained $20 \mathrm{~g}$ LAC whey protein (Murray Goulburn, Melbourne, Vic, Australia), and in the control condition, each drink contained $20 \mathrm{~g}$ calcium caseinate (Fonterra Ingredients Innovation, Palmerston North, New Zealand) (Table 1). The chocolate drink in the LAC condition contained $2.6 \mathrm{~g} / 100 \mathrm{~g}$ TRP (Trp:LNAA ratio of 7.6), whereas the chocolate drink in the control condition contained $1.3 \mathrm{~g} / 100 \mathrm{~g}$ TRP (Trp:LNAA ratio of 4 ). The chocolate drinks did not differ on a range of palatability ratings (SW, savoury (SA), filling, satisfying and tasty) (largest $F(1)=3.456, \quad P=0.075$ ). On both the experimental days, participants were instructed to consume their snack and lunch foods within $15 \mathrm{~min}$. Participants were not permitted to consume other foods during the test day until leaving the research laboratory.

\section{Stress task}

A mental arithmetic task, performed during noise-distraction stimulation, was used as an experimental stressor ${ }^{(26,27)}$. Participants were presented with fifteen 1-min trials in

Table 1. Composition and amino acid profile of the chocolate drinks used in the $\alpha$-lactalbumin (LAC) and casein (CAS) diets

\begin{tabular}{lcc}
\hline & LAC & CAS \\
\hline Composition (g) & 20 & \\
LAC & 0 & 20 \\
Caseinate & 40 & 40 \\
Chocolate powder & 10 & 10 \\
Granulated sugar & 400 & 400 \\
Water & 697.94 & 697.94 \\
Energy (kJ) & 1.2 & \\
Amino acid profile (amount per $20 \mathrm{~g}$ protein) (g) & 1.06 \\
Ile & 2.74 & 1.88 \\
Leu & 0.82 & 1.04 \\
Phe & 0.52 & 0.26 \\
Trp & 1.2 & 1.34 \\
Val & 0.9 & 1.16 \\
Tyr & 7.60 & 4.00 \\
Trp:LNAA (weight \%) & & \\
\hline
\end{tabular}

LNAA, large neutral amino acids. which they had to solve multiple choice mental arithmetic questions on a computer screen. Simultaneously with the multiple choice questions, three different levels of industrial noise $(65,70$ or $80 \mathrm{~dB})$ were delivered through headphones. In every trial, participants were instructed to correctly solve a minimum target number of calculations. Participants were informed that the intensity of the noise would be influenced by the quality of their performance. If they failed to solve the target number correctly, the noise level would automatically increase during the next trial. If participants solved the target number correctly, they could self-select the noise level. The target number was set so that participants automatically failed on every trial; hence, they were continuously unable to volitionally select the noise level for subsequent trials.

\section{Mood}

Mood was measured using the Positive and Negative Affect Schedule ${ }^{(36)}$. The paper-and-pencil version of the Positive and Negative Affect Schedule consists of ten positive affects (PA; e.g. interested, excited and alert) and ten negative affects (NA; e.g. distressed, ashamed and nervous). Participants were asked to rate items on a scale from $1=$ 'not at all' to $5=$ 'extremely' depending on their current mood state.

\section{Appetite}

Subjective sensations of appetite were monitored using a computerised visual analogue scale procedure programmed in E-prime 1.2 software ${ }^{(37)}$. Visual analogue scales were used to assess hunger (i.e. how hungry do you feel now?), fullness, desire to eat and appetite for something SW and something SA. Participants responded by placing the cursor on a line that was $100 \mathrm{~mm}$ in length. Participants used the mouse to move a centred cursor along the line, and once satisfied with the position of the cursor, they clicked on the mouse to indicate their answer. Each line was anchored on the left side with 'not at all' and on the right side with 'extremely'.

\section{Food hedonics}

Food hedonics were examined using a novel experimental procedure to measure liking, wanting and food preference in response to photographic images of food ${ }^{(38-40)}$. Sixteen food stimuli were presented on a computer screen. The foods were organised into separate categories of SW, SA, high fat and low fat. For a detailed description of the liking and wanting procedure, see Finlayson et al. ${ }^{(38)}$.

Liking was defined as the anticipated pleasure derived from tasting a food, and was measured by presenting each food stimulus one at a time, and participants rated their perceived pleasantness on a 100-unit visual analogue scale anchored at each end with 'not at all' to 'extremely' combined with the statement 'How pleasant would it be to taste this food now?' Participants were asked to move a centred cursor along the line to indicate their response. Mean liking ratings for each food category were calculated ${ }^{(38)}$.

Food preference was defined as the selection of a food over relevant alternatives at the point of choice, and was measured by presenting the food stimuli in pairs, and participants were 
instructed to select the food they 'would most like to eat now' via keypress on the keyboard. This forced choice task consisted of a total of ninety-six pairs of combinations, from which the frequency of selections per category was calculated $^{(38)}$.

The computer procedure also records reaction time latencies during the forced choice task to measure implicit wanting ${ }^{(39)}$, but these data were not used in the present study.

\section{Statistical analysis}

Statistical analyses were conducted on data from the twenty-five participants who completed the study, since this was not an intention to treat analysis. Independent sample $t$ tests were performed on key demographic variables to determine whether the groups were similar in this regard (SPSS 16.0 for Windows (Chicago, IL, USA)). Testing for normal distribution was conducted using the Shapiro-Wilk test of normality. Since all data were not normally distributed, logarithmic transformations (log-10) were applied. Transformations were unsuccessful, and as a result, the implementation of non-parametric tests was required. Nevertheless, the research question was focused on investigating the interactions between intervention, group and stress, a goal that cannot be achieved with non-parametric tests. As a solution, data were analysed with parametric and non-parametric test statistics, resulting in the same conclusion regarding the significance of effects. For clarity, only parametric test statistics were reported.

The main research question was analysed by means of univariate ANOVA by using the general linear model with one between-subjects factor Group (HA $v$. LA) and two within-subjects factors Diet (LAC $v$. CAS) and Time (baseline $v$. pre-stress) or Stress (pre-stress $v$. post-stress) as withinsubjects factor on the several dependent measures. To investigate the effects of the intervention and the effects of stress separately, we included Time $(t 1 v . t 2 v . t 3)$ in the first analysis and Stress (pre-stress, $t 3 v$. post-stress, $t 4$ ) in the second analysis as within-subjects factor. Greenhouse-Geisser corrected $P$ values are reported when the sphericity assumption was not met.

In the initial analyses, BMI was included as a covariate, although omitted from the final analyses due to its insignificance (largest $F(1)=1 \cdot 724, P=0.203$ ). All statistics were evaluated two-tailed at a significance level of $5 \%$. Data are reported as means and standard deviations.

\section{Results \\ Demographic characteristics}

No significant differences existed between the LA and HA groups regarding age, BMI and three factor eating questionnaire eating behaviour traits (Table 2). As anticipated, the HA group had a significantly higher average anxiety score compared with the LA group, $t(23)=-6.998, P<0.0001$.

\section{Mood}

In the morning period, before the stress task, repeatedmeasures ANOVA revealed no interactions and no main effect of Diet on the positive and negative subscales of the Positive and Negative Affect Schedule (Fig. 2). A main effect of Group $(F(1,23)=6 \cdot 232, P=0 \cdot 020)$ was revealed on PA. Further analyses showed that the HA group reported a significantly lower positive mood compared with the LA group after the snack $(F(1,23)=7 \cdot 250, P=0.013)$ and after the lunch $(F(1,23)=5.163, P=0.033)$ independent of diet. There was a main effect of Time $(F(2,22)=3 \cdot 539$, $P=0.046)$ on NA, reflecting a decrease in NA after lunch compared with the first morning measurement $(F(1,23)=7 \cdot 085, P=0 \cdot 014)$.

\section{Stress task and mood}

There were no significant interactions after the stress task. A significant main effect of Stress on mood was revealed, represented by a significant decrease in PA scores $(F(1,23)=13.077, P=0.001)$, whereas NA scores increased $(F(1,23)=18.435, P<0.0001)$ after the stress task. These results demonstrated that the stress task was effective at debilitating mood. There were no significant main effects of Diet on PA or NA after stress, nor was there a significant Group effect on NA. However, there was a main effect of Group on PA $(F(1,23)=6.688, \quad P=0 \cdot 017)$, demonstrating that the HA group reported a lower positive mood regardless of diet.

\section{Appetite}

Before the stress task, there were no significant interactions or significant main effects of Diet on subjective sensations of appetite or a main effect of Diet (Fig. 3). However, main effects of Group and Time were revealed on the subscales

Table 2. Demographic characteristics of the low- $(L A)$ and high-anxiety $(H A)$ groups (Mean values and standard deviations)

\begin{tabular}{|c|c|c|c|c|c|c|}
\hline & \multicolumn{2}{|c|}{$\begin{array}{c}\text { LA } n 12 \\
\text { (eleven } \mathrm{F} \text { and one M) }\end{array}$} & \multicolumn{2}{|c|}{$\begin{array}{c}\text { HA } n 13 \\
\text { (eleven } \mathrm{F} \text { and two M) }\end{array}$} & \multirow{2}{*}{$\begin{array}{l}\text { Test statistic } \\
t(23)\end{array}$} & \multirow[b]{2}{*}{$P$} \\
\hline & Mean & SD & Mean & SD & & \\
\hline Age (years) & $21 \cdot 0$ & 1.7 & 21.5 & $5 \cdot 7$ & -0.314 & 0.76 \\
\hline BMI $\left(\mathrm{kg} / \mathrm{m}^{2}\right)$ & 23.4 & 2.5 & 22.9 & $3 \cdot 0$ & 0.455 & 0.65 \\
\hline \multicolumn{7}{|l|}{ TFEQ } \\
\hline Restraint & 9.6 & $3 \cdot 8$ & $7 \cdot 0$ & $5 \cdot 2$ & 1.41 & 0.17 \\
\hline Hunger & 5.4 & $2 \cdot 8$ & 6.5 & 2.5 & -1.06 & 0.30 \\
\hline Disinhibition & 4.7 & $2 \cdot 6$ & $6 \cdot 2$ & 3.4 & -1.22 & 0.23 \\
\hline STAI & 30.4 & $4 \cdot 8$ & 45.5 & 5.9 & -6.99 & $<0.001^{*}$ \\
\hline
\end{tabular}

F, female; M, male; TFEQ, three-factor eating questionnaire; STAI, state-trait anxiety questionnaire; $t$, $t$ test.

* Groups differ exclusively in trait anxiety as measured by the STAI. 

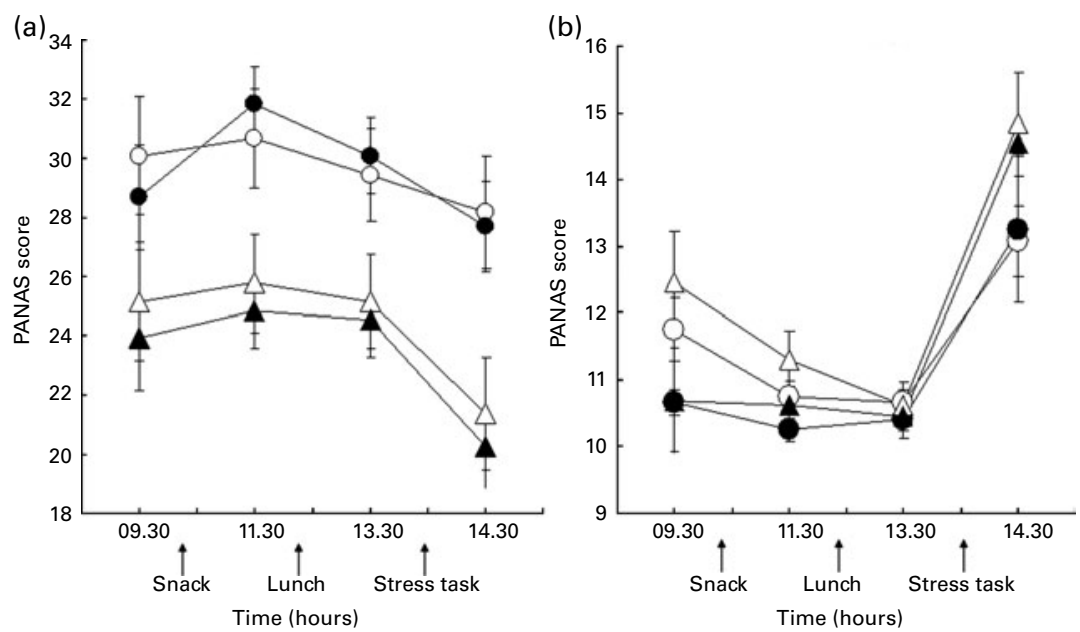

Fig. 2. Temporal profile of positive (a) and negative (b) affects measured by the Positive and Negative Affect Schedule (PANAS) as a function of group and diet. $O$, Low-trait anxiety group (LA) - casein (CAS) diet; $\bullet$, LA - $\alpha$-lactalbumin (LAC) diet; $\triangle$, high-trait anxiety group (HA) - CAS diet; $\mathbf{\Lambda}$, HA - LAC diet.

hunger, fullness and desire to eat. The HA group reported feeling hungrier, less full and a stronger desire to eat at baseline and after snack (lowest $F(1,23)=4 \cdot 711, P=0.041$ ) compared with the LA group. Feelings of hunger and desire to eat increased, while fullness decreased after baseline and snack, and the reverse, experiencing less sensations of appetite, occurred after lunch (lowest $F(2,22)=14 \cdot 101, P<0 \cdot 0001)$.

\section{Stress task and appetite}

After stress, no significant interactions or significant main effects of Diet or Group were revealed. There was a main effect of Stress for subjective sensations of appetite. Specifically, participants reported an increase in hunger and stronger desire to eat, and lower fullness after the stress task (lowest $F(1,23)=17.055, \quad P<0.0001)$. Furthermore, the desire for SW $(F(1,23)=33.440, P<0.0001)$ and SA foods $(F(1,23)=15.974, P=0.001)$ increased after the stress task. Importantly, these effects occurred independent of diet or group. Although the increase in appetite could be partly explained by the demands of the stressful challenge, some or all of the increase in appetite could simply reflect the natural rise in hunger during the post-absorptive period.

\section{Food hedonics}

Data from the food hedonic task were analysed on the basis of four taste/nutrient combinations (high fat SA, high fat SW, low fat SA and low fat SW) and SA or SW categories based on the aggregate of high- and low-fat categories. The results from the SW categorisation were reported since the hypotheses focused more on the sensory characteristic of sweetness, rather than on the nutrient fat.

\section{Stress task and food hedonics}

Liking. A significant main effect of Stress $(F(1,23)=10.641, P=0.003)$ on liking for SW foods was found, indicating that liking of $\mathrm{SW}$ foods increased after stress exposure (Fig. 4). Moreover, univariate analysis revealed a significant Diet $\times$ Group interaction effect on the liking subtask $(F(1,23)=7 \cdot 356, P=0 \cdot 012)$. Further analysis
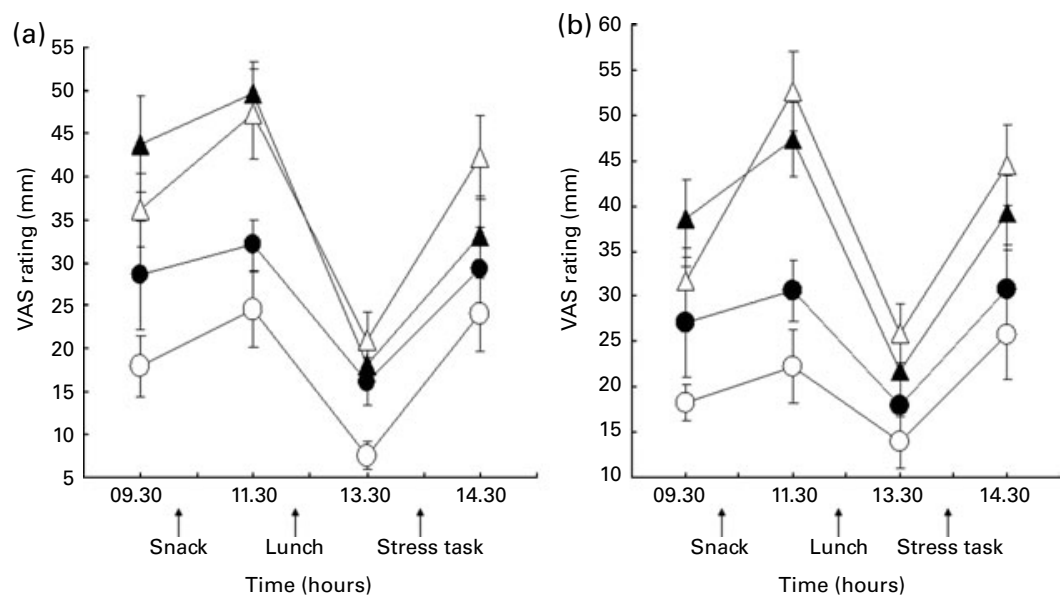

Fig. 3. Temporal profile of subjective sensations of hunger (a) and desire to eat (b) as a function of group and diet. $O$, Low-trait anxiety group (LA) - casein (CAS) diet; $\bullet$, LA - $\alpha$-lactalbumin (LAC) diet; $\triangle$, high-trait anxiety group (HA) - CAS diet; $\boldsymbol{\Delta}$, HA - LAC diet. VAS, visual analogues scales. 
(a)

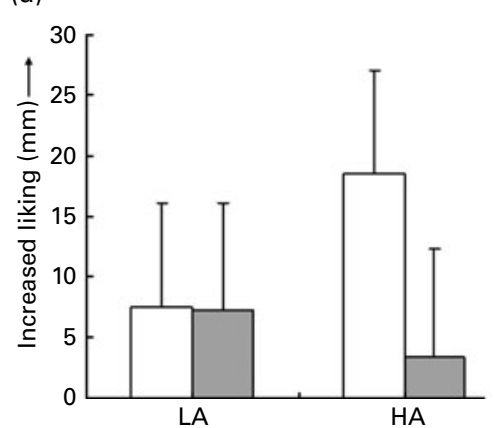

(b)

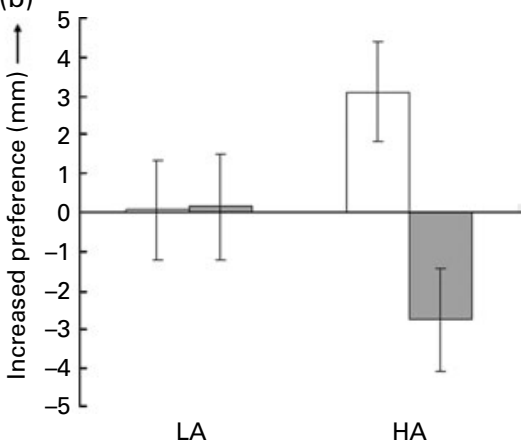

Fig. 4. Mean change ( $t 3$, pre stress; $t 4$, post stress) in mm for liking (a) and preference for sweet foods (b) as a function of diet and group. LA, low-trait anxiety group; HA, high-trait anxiety group. $\square$, Casein; $\square, \alpha$-lactalbumin.

revealed a significant increase in liking for SW foods immediately after the stress task during the CAS dietary manipulation compared with the LAC manipulation for the HA group only $(t(12)=-4 \cdot 251, P=0.001)$. The HA group reported a $49 \%$ increase in liking for SW foods after the stress task on the CAS diet, while reporting only a $9.3 \%$ increase in liking for SW foods on the LAC test day. No significant main or interaction effects were found on the SA category.

Food preference. Univariate analysis revealed a significant Diet $\times$ Group $\times$ Stress interaction effect on preference for SW foods $(F(1,23)=7.091, \quad P=0.014)$. Immediately after the stress task, the HA group reported an increase in SW food preference $(10 \%)$ on the CAS test day compared with a decreased preference for SW foods $(9 \%)$ on the LAC test day $(t(12)=3.336, P=0.006)$, while this was not found for the LA group $(t(11)=-0.063, P=0.951)$.

\section{Discussion}

The present study examined the acute effects of LAC on subjective mood and eating behaviour in individuals with low- and high-trait anxiety before and after exposure to a stress task. It was expected that any detrimental effects of acute stress on mood and eating behaviour in high-trait anxiety individuals would be alleviated by 5-HTergic augmentation via LAC. Nonetheless, dietary supplemented LAC did not exert any immediate effects on stress, mood or appetite ratings. In contrast, LAC significantly exerted a selective effect on liking and preference for SW foods, which occurred only in high-trait anxiety individuals.

Numerous previous studies from different research groups reported a $50-130 \%$ rise in plasma Trp/LNAA after the same dose of comparable LAC protein sources ${ }^{(26-28,31,32)}$. Since a $50 \%$ increase in plasma Trp/LNAA is believed to cause meaningful changes in brain TRP and $5-\mathrm{HT}^{(26,41-45)}$, it seems justified to assume that after the LAC supplement, more TRP was available for uptake in the brain in the present study. In addition, LAC was expected to enhance brain 5-HT function, thereby preventing subsequent detrimental effects of stress exposure on mood and eating behaviour, particularly in high-trait anxiety individuals.

Data revealed debilitated mood after acute stress exposure, thereby confirming successful stress induction by the stress task. This was characterised by a decrease in PA and an increase in NA. This effect of the stress task on affective changes is comparable with physiological changes including cardiovascular (pulse rate, skin conductance and blood pressure) and endocrine (cortisol) stress responses after this particular task ${ }^{(26,27,46,47)}$. The stress task also increased subjective appetite sensations, a finding that has often been demonstrated under mood-inducing stressful circumstances $^{(23-25,48-51)}$.

It was hypothesised that high-trait anxiety individuals would report greater mood decline after the stress task than low-trait anxiety individuals, and that LAC would prevent such a stress-induced deterioration of mood in high-trait anxiety individuals. This expectation was based on the assumption that high-trait anxiety relates to chronic stress and promotes brain 5-HTergic vulnerability. It was proposed that hypersecretion of cortisol and/or increased breakdown of 5-HT may alter brain 5-HT responsiveness due to compensatory receptor sensitisation ${ }^{(18-21)}$. Consequently, LAC was expected to improve brain 5-HT function and, accordingly, stress coping and mood most strongly in individuals with high-trait anxiety scores.

However, the negative effects of stress on mood did not differentiate between the low- and high-trait anxiety groups, and this was not moderated by the acute LAC manipulation. These findings seem to conflict with previous data of Markus et al. ${ }^{(26)}$ who observed a protective effect on stressinduced changes in mood and cortisol stress responses, exclusively in individuals scoring high on neuroticism compared with individuals with low neuroticism scores. There are several potential explanations for the conflicting findings. Firstly, the present study included a rather general positive or negative mood measurement (Positive and Negative Affect Schedule), whereas Markus et al. ${ }^{(26)}$ used five subscales of the POMS, which reveals mood changes in several specific directions, including depression. Secondly, in the present study, chronic stress vulnerability was operationalised on the basis of trait anxiety, and participants were separated by a median split in trait anxiety scores. This resulted in two relatively small groups with upper $(\mathrm{STAI}=45)$ or lower $(\mathrm{STAI}=30)$ normal range scores. Markus et al. ${ }^{(26)}$ operationalised chronic stress on the basis of extreme neuroticism scores, and subsequently included two large groups either selected from the highest quartile of inadequacy scores (26) or lowest quartile of inadequacy scores (4). Even though the high-trait anxiety group in the present study reported lower positive baseline mood than the low-trait anxiety group, 
using a median split difference on trait anxiety scores may still be less sufficient to distinguish chronic stress and related 5-HTergic vulnerability.

While a stress-induced increase in the desire for SW foods has been reported previously ${ }^{(48-51)}$, these are the first data to reveal that the effect is moderated by an acute dietary manipulation. Theoretically, this stress-induced preference for SW foods may relate to a stress-induced deterioration in mood and hence brain $5-\mathrm{HT}^{(18,19,52)}$. In addition, such changes in 5-HT are believed to alter 5-HT function in the ventromedial hypothalamus that selectively influences intake of SW carbohydrate-rich foods ${ }^{(53,54)}$. Based on the assumption that 5-HT is involved in moderating stress and mood, and that trait anxiety is associated with chronic stress-induced 5-HT vulnerability, it was expected that the high-trait anxiety individuals would be sensitive to the combination of LAC and stress exposure. The LAC manipulation prevented an increase in liking and preference for SW foods after stress exclusively in high-trait anxiety individuals. These data support the concept that overconsumption can be driven by non-homeostatic processes. Indeed, it strongly implicates a role for hedonic (i.e. sensorily driven) processes of overconsumption in conjunction with exposure to stress ${ }^{(48,49)}$.

As mentioned previously, the present study did not measure variations in plasma Trp:LNAA ratio to verify dietary effects. However, based on previous research ${ }^{(26-28,31,32)}$, it is assumed that the Trp:LNAA ratio was augmented in the present study. Nevertheless, two confounding factors might have influenced the magnitude of changes in the plasma Trp:LNAA ratio. Firstly, participants were allowed to eat their regular breakfast on both the test days, which might have influenced the baseline Trp:LNAA ratio and/or the magnitude of treatment effect on these amino acids. Nevertheless, participants consumed an identical breakfast on both the test days in a crossover design, which leaves it highly unlikely that this was a meaningful confounding factor. Secondly, the study did not control for the menstrual cycle of female participants. Both 5-HT levels as well as mood and eating behaviour correlate with the menstrual cycle ${ }^{(55-57)}$. Specifically, during the follicular phase, women have higher 5-HT levels and experience more positive mood, less appetite and less desire to eat SW foods compared with the luteal phase of the cycle $^{(57)}$. Ideally, test days should have been separated by 4 weeks to allow for the control of the menstrual cycle. No information was collected on menstrual cycle - hence, it was not possible to account for menstrual cycle variation.

A final consideration refers to the stress paradigm used in the present study. Although this particular stress task has already been shown to cause psychological, cardiovascular and endocrine stress responses ${ }^{(26,27,46,47)}$, only psychological (mood) changes were used to confirm stress induction in the present study.

In conclusion, tryptophan-rich LAC protein prevented a stress-induced increase in liking and preference for SW foods exclusively in individuals with high-trait anxiety scores. This effect occurred despite there being no impact of diet on subjective mood or overall appetite. Since LAC is found to increase brain 5-HT, these data suggest greater brain 5-HTergic vulnerability for stress-induced eating behaviour particularly in high-trait anxious individuals. The beneficial effects of brain 5-HT manipulation in high-trait anxiety individuals are expected to become stronger, and may even improve a stress-induced mood decline, when including a more rigorous stressful task. In addition, clinical or subclinical individuals with more severe brain 5-HTergic vulnerabilities should be included in further studies to explore whether LAC could have therapeutic potential in 5-HT-related disorders such as depression or eating disturbances.

\section{Acknowledgements}

LAC and CAS protein were provided, respectively, by Murray Goulburn and Fonterra Ingredients Innovation, whose support is gratefully acknowledged. The present research received no specific grant from any funding agency in the public, commercial or not-for-profit sectors. Authors E. V. and N. A. K. designed the study. E. V. wrote the manuscript and conducted the statistical analysis under supervision of N. A. K., G. F., J. B. and C. R. M. reviewed the manuscript. All authors contributed to and have approved the final manuscript. The authors declare no conflict of interest.

\section{References}

1. Carrasco JL \& Sandner C (2005) Clinical effects of pharmacological variations in selective serotonin reuptake inhibitors: an overview. Int J Clin Pract 59, 1428-1434.

2. Edwards JG \& Anderson I (1999) Systematic review and guide to selection of selective serotonin reuptake inhibitors. Drugs 57, 507-533.

3. Thase ME, Trivedi MH \& Rush AJ (1995) MAOIs in the contemporary treatment of depression. Neuropsychopharmacology 12, 185-219.

4. To SE, Zepf RA \& Woods AG (2005) The symptoms, neurobiology, and current pharmacological treatment of depression. J Neurosci Nurs 37, 102-107.

5. Halford JC, Harrold JA, Lawton CL, et al. (2005) Serotonin (5-HT) drugs: effects on appetite expression and use for the treatment of obesity. Curr Drug Targets 6, 201-213.

6. Leibowitz SF \& Alexander JT (1998) Hypothalamic serotonin in control of eating behavior, meal size, and body weight. Biol Psychiatry 44, 851-864.

7. Wurtman J, Wurtman R, Mark S, et al. (1985) D-Fenfluramine selectively suppresses carbohydrate snacking by obese subjects. Int J Eat Disord 4, 89-99.

8. McAllister-Williams RH, Ferrier IN \& Young AH (1998) Mood and neuropsychological function in depression: the role of corticosteroids and serotonin. Psychol Med 28, 573-584.

9. Simansky KJ (1996) Serotonergic control of the organization of feeding and satiety. Behav Brain Res 73, 37-42.

10. Brewerton TD (1995) Toward a unified theory of serotonin dysregulation in eating and related disorders. Psychoneuroendocrinology 20, 561-590.

11. Riedel WJ, Klaassen T \& Schmitt JA (2002) Tryptophan, mood, and cognitive function. Brain Behav Immun 16, 581-589.

12. Bell C, Abrams J \& Nutt D (2001) Tryptophan depletion and its implications for psychiatry. Br J Psychiatry 178, 399-405.

13. Bell CJ, Hood SD \& Nutt DJ (2005) Acute tryptophan depletion. Part II: clinical effects and implications. Aust N Z J Psychiatry 39, 565-574.

14. Booij L, Van der Does AJ \& Riedel WJ (2003) Monoamine depletion in psychiatric and healthy populations: review. Mol Psychiatry 8, 951-973.

15. Booij L, Van der Does AJ, Haffmans PM, et al. (2005) The effects of high-dose and low-dose tryptophan depletion on 
mood and cognitive functions of remitted depressed patients. J Psychopharmacol 19, 267-275.

16. Van der Does AJ (2001) The effects of tryptophan depletion on mood and psychiatric symptoms. J Affect Disord 64, 107-119.

17. Weltzin TE, Fernstrom MH, Fernstrom JD, et al. (1995) Acute tryptophan depletion and increased food intake and irritability in bulimia nervosa. Am J Psychiatry 152, 1668-1671.

18. Firk C \& Markus CR (2007) Review: serotonin by stress interaction: a susceptibility factor for the development of depression? J Psychopharmacol 21, 538-544.

19. Jans LA, Riedel WJ, Markus CR, et al. (2007) Serotonergic vulnerability and depression: assumptions, experimental evidence and implications. Mol Psychiatry 12, 522-543.

20. Markus CR, Panhuysen G, Tuiten A, et al. (1998) Does carbohydrate-rich, protein-poor food prevent a deterioration of mood and cognitive performance of stress-prone subjects when subjected to a stressful task? Appetite 31, 49-65.

21. Van Praag HM (1996) Faulty cortisol/serotonin interplay, psychopathological and biological characterisation of a new, hypothetical depression subtype (SeCA depression). Psychiatry Res 65, 143-157.

22. Felsten G (2004) Stress reactivity and vulnerability to depressed mood in college students. Pers Indiv Differ 36, 789-800.

23. Oliver G, Wardle J \& Gibson EL (2000) Stress and food choice: a laboratory study. Psychosom Med 62, 853-865.

24. Zellner DA, Loaiza S, Gonzalez Z, et al. (2006) Food selection changes under stress. Physiol Behav 87, 789-793.

25. Epel E, Lapidus R, McEwen B, et al. (2001) Stress may add bite to appetite in women: a laboratory study of stress-induced cortisol and eating behavior. Psychoneuroendocrinology 26, 37-49.

26. Markus CR, Olivier B, Panhuysen GE, et al. (2000) The bovine protein alpha-lactalbumin increases the plasma ratio of tryptophan to the other large neutral amino acids, and in vulnerable subjects raises brain serotonin activity, reduces cortisol concentration, and improves mood under stress. Am J Clin Nutr 71, 1536-1544.

27. Markus CR, Olivier B \& de Haan EH (2002) Whey protein rich in alpha-lactalbumin increases the ratio of plasma tryptophan to the sum of the other large neutral amino acids and improves cognitive performance in stress-vulnerable subjects. Am J Clin Nutr 75, 1051-1056.

28. Markus CR, Jonkman LM, Lammers JH, et al. (2005) Evening intake of alpha-lactalbumin increases plasma tryptophan availability and improves morning alertness and brain measures of attention. Am J Clin Nutr 81, 1026-1033.

29. Heine W, Radke M, Wutzke KD, et al. (1996) Alpha-lactalbumin-enriched low-protein infant formulas: a comparison to breast milk feeding. Acta Paediatr 85, 1024-1028.

30. Lehnert H \& Wurtman RJ (1993) Amino acid control of neurotransmitter synthesis and release: physiological and clinical implications. Psychother Psychosom 60, 18-32.

31. Merens W, Booij L, Markus R, et al. (2005) The effects of a diet enriched with alpha-lactalbumin on mood and cortisol response in unmedicated recovered depressed subjects and controls. $\mathrm{Br} \mathrm{J}$ Nutr 94, 415-422.

32. Beulens JW, Bindels JG, de Graaf C, et al. (2004) Alpha-lactalbumin combined with a regular diet increases plasma Trp-LNAA ratio. Physiol Behav 81, 585-593.

33. Spielberger CD, Gorsuch RC \& Lushene RE (1970) Manual for the State Trait Anxiety Inventory. Palo Alto, CA: Consulting Psychologists Press.

34. Bieling PJ \& Alden LE (1997) The consequences of perfectionism for patients with social phobia. Br J Clin Psychol 36, Pt 3, 387-395.

35. Bieling PJ, Antony MM \& Swinson RP (1997) The statetrait anxiety inventory, trait version: structure and content re-examined. Behav Res Ther 36, 777-788.
36. Watson D, Clark LA \& Tellegen A (1988) Development and validation of brief measures of positive and negative affect: the PANAS scales. J Pers Soc Psychol 54, 1063-1070.

37. Schneider W, Eschman A \& Zuccolotto A (2002) E-Prime User's Guide. Pittsburgh, PA: Psychology Software Tools.

38. Finlayson G, King N \& Blundell JE (2007) Is it possible to dissociate 'liking' and 'wanting' for foods in humans? A novel experimental procedure. Physiol Behav 90, 36-42.

39. Finlayson G, King N \& Blundell J (2008) The role of implicit wanting in relation to explicit liking and wanting for food: implications for appetite control. Appetite 50, 120-127.

40. Finlayson G, King N \& Blundell JE (2007) Liking vs. wanting food: importance for human appetite control and weight regulation. Neurosci Biobehav Rev 31, 987-1002.

41. Fernstrom JD (1999) Aromatic amino acids and monoamine synthesis in the central nervous system: influence of the diet. J Nutr Biochem 1, 508-517.

42. Fernstrom JD \& Wurtman RJ (1971) Brain serotonin content: increase following ingestion of carbohydrate diet. Science 174, $1023-1025$.

43. Fernstrom JD \& Wurtman RJ (1972) Brain serotonin content: physiological regulation by plasma neutral amino acids. Science 178, 414-416.

44. Curzon G (1985) Effects of food intake on brain transmitter amine precursors and amine synthesis. Psychopharmacology and Food, pp. 59-70 [M Sandler and T Silverstone, editors]. Oxford: Oxford University Press.

45. Orosco M, Rouch C, Beslot F, et al. (2004) Alpha-lactalbuminenriched diets enhance serotonin release and induce anxiolytic and rewarding effects in the rat. Behav Brain Res 148, 1-10.

46. Peters ML, Godaert GL, Ballieux RE, et al. (1998) Cardiovascular and endocrine responses to experimental stress: effects of mental effort and controllability. Psychoneuroendocrinology 23, $1-17$.

47. Markus CR, Panhuysen G, Tuiten A, et al. (1998) Does carbohydrate-rich, protein-poor food prevent a deterioration of mood and cognitive performance of stress-prone subjects when subjected to a stressful task? Appetite 31, 49-65.

48. Newman E, O'Connor DB \& Conner M (2008) Attentional biases for food stimuli in external eaters: possible mechanism for stress-induced eating? Appetite 51, 339-342.

49. Conner M, Fitter M \& Fletcher W (1999) Stress and snacking: a diary study of daily hassles and between-meal snacking. Psychol Health 14, 51-63.

50. Greeno CG \& Wing RR (1994) Stress-induced eating. Psychol Bull 115, 444-464.

51. Rutters F, Nieuwenhuizen AG, Lemmens SG, et al. (2009) Acute stress-related changes in eating in the absence of hunger. Obesity (Silver Spring) 17, 72-77.

52. Markus CR (2008) Dietary amino acids and brain serotonin function; implications for stress-related affective changes. Neuromolecular Med 10, 247-258.

53. Blundell JE (1984) Serotonin and appetite. Neuropharmacology 23, 1537-1551.

54. Leibowitz SF \& Alexander JT (1998) Hypothalamic serotonin in control of eating behavior, meal size, and body weight. Biol Psychiatry 44, 851-864.

55. Dimmock PW, Wyatt KM, Jones PW, et al. (2000) Efficacy of selective serotonin-reuptake inhibitors in premenstrual syndrome: a systematic review. Lancet 356, 1131-1136.

56. Dye L \& Blundell JE (1997) Menstrual cycle and appetite control: implications for weight regulation. Hum Reprod 12, 1142-1151.

57. Hindberg I \& Naesh O (1992) Serotonin concentrations in plasma and variations during the menstrual cycle. Clin Chem 38, 2087-2089. 